\title{
musculoesqueléticos
}

\section{en odontólogos}

\section{Autores:}

Karla Lizbeth Fimbres Salazar. Licenciada en Enfermería. División de Ciencias Biológicas y de la Salud, Departamento de Enfermería de la Universidad de Sonora. E-mail: karla.fimbress@gmail.com

Julio Alfredo García Puga. Enfermero, Doctor en Ciencias Sociales. División de Ciencias Biológicas y de la Salud, Departamento de Enfermería de la Universidad de Sonora. E-mail: alfredogarcia@enfermeria.uson.mx

Rosa María Tinajero González. Enfermera, Doctora en Educación. División de Ciencias Biológicas y de la Salud, Departamento de Enfermería de la Universidad de Sonora. E-mail: rosa.tinajero@unison.mx

Rosa Elena Salazar Rubial. Enfermera, Doctora en Educación. División de Ciencias Biológicas y de la Salud, Departamento de Enfermería de la Universidad de Sonora. E-mail: rsalazar@enfermeria.uson.mx

María Olga Quintana Zavala. Enfermera, Doctora en Enfermería. División de Ciencias Biológicas y de la Salud, Departamento de Enfermería de la Universidad de Sonora. E-mail: olga_quintana@enfermeria.uson.mx

*Investigación derivada de tesis de pregrado.

\section{> Resumen}

El objetivo de la presente investigación fue identificar trastornos musculoesqueléticos en odontólogos de una clínica dental en Hermosillo, Sonora. Material y método. Se realizó un estudio cuantitativo, no experimental, transversal y observacional, el muestreo fue no probabilístico y la muestra estuvo constituida por 30 sujetos. Se utilizaron los instrumentos Cuestionario Estandarizado Nórdico y el método Rapid Entire Body Assessment. Resultados. Se obtuvo un nivel de riesgo medio (70\%) y con una necesidad de implementar acciones del $70 \%$. Al evaluar la sintomatología de cada zona anatómica, durante la práctica odontológica, se observó un predomino de sintomatología en cuello (70\%), región dorsal o lumbar (63,3\%), hombros (46,7\%), el lado derecho presenta más alteraciones (36,7\%), manos o muñecas $(46,7 \%)$ y mayor frecuencia en mano o muñeca derecha (33,3\%), en codos o antebrazos la mayoría señala no tener molestia (90\%). Conclusiones. De los trastornos músculo-esqueléticos en odontólogos sobresalen molestias en cuello, región dorsal o lumbar, hombros, manos y muñecas, con mayor alteración en la parte derecha. Desde la enfermería laboral se deben proponer acciones para disminuir el riesgo.

, Palabras claves: Enfermedades Musculoesqueléticas, Odontología, Enfermería (DeCS). 
disorders in

odontologists

\section{> Abstract}

The objective of this research was to identify the musculoskeletal disorders in odontologists from a dental clinic in Hermosillo, Sonora. Material and Method. A quantitative study was conducted, not experimental, transversal and observational, sampling was non-probabilistic. The sample consisted of 30 subjects. The instruments used were the Nordic standardized questionnaire with a Cronbach's alpha of 0.83 and the Rapid Entire Body Assessment method. Results. The study got a mean level of risk (70\%) with the necessity of implementing actions of $70 \%$. Assessing symptoms of each anatomical region, during the Dental Practice, it was observed a predominance of symptoms in the neck (70\%), dorsal or lumbar region (63.3\%), shoulders (46.7\%). Also, right side presents more alterations (36.7\%), hands or wrists (46.7\%) and frequently continues right hand or wrist (33.3\%). Most subjects indicate do not have any hassle (90\%). Conclusions. Among the musculoskeletal disorders in dentists, outstands neck discomfort, thoracic or lumbar region, shoulders, hands and wrists, with more alterations on the right side. Nursing should propose actions in order to reduce the risk.

, Keywords: Musculoskeletal Diseases, Dentistry, Nursing (DeCS). 


\section{) Introducción}

La Odontología es una profesión y disciplina del área de las ciencias de la salud que tiene por objeto de estudio la salud bucal de los seres humanos en las diferentes etapas de su desarrollo, dependiendo de sus necesidades a nivel individual y colectivo (1). El personal que practica esta actividad se ve sometido a una serie de posturas que pueden condicionar lesiones en su aparato locomotor, por ello, se requiere que se realice prevención de dichas lesiones y es donde la ergonomía como disciplina científica estudia el trabajo humano, aportando principios básicos para la organización de las tareas, principios que, aplicados a la clínica odontológica, dan como resultado una racionalización de los procedimientos obligatorios, una simplificación de las rutinas en el consultorio y una significativa economía de tiempos (2).

Sin embargo, el personal continuará expuesto a diversos factores de riesgo, entendidos como cualquier rasgo, característica o exposición de un individuo que aumente su probabilidad de sufrir una enfermedad o lesión (3), por lo que es necesario mantener una vigilancia contante de estos aspectos.

Los odontólogos debido a su trabajo, son proclives a sufrir problemas en el aparato locomotor, situación que en ocasiones puede limitar su rendimiento laboral. Estos problemas pueden ocasionar desde una ligera sintomatología músculoesquelética, hasta la incapacidad del profesional (en casos severos), poniendo en riesgo no sólo su salud, sino también mermando severamente el rendimiento profesional, con ello se afecta la institución donde labora, disminuyendo la calidad del servicio que ofrece (4).

Por tanto, la práctica de la odontología es una de las profesiones que presenta mayor riesgo de de- sarrollar Trastornos Musculoesqueléticos (TME) relacionados con el trabajo debido a la adopción de posturas, que pueden ser inadecuadas, durante la actividad clínica odontológica y movimientos altamente repetitivos que demandan algún componente de fuerza y factores físicos o psicosociales asociados (5).

Dentro de las principales lesiones reportadas en odontólogos se han encontrado tendinitis (27,5\%), pericapsulitis de hombro (27,5\%), síndrome del túnel del carpio (27,5\%) y síndrome cervical por tensión (60,8\%). Las causas de estas lesiones derivan de la exposición de los odontólogos a los factores de riesgo, las más significativas son posturas forzadas, movimientos repetitivos, la misma posición por un descanso y el mal diseño del puesto de trabajo (6).

Por otro lado, se ha develado una prevalencia de signos y síntomas de trastornos de la mano del 33,0\% que pueden estar directamente relacionados con el ejercicio de la profesión odontológica cuyo predominio fue en mano derecha (92,2\%), destacándose síntomas de adormecimiento de la mano (26,2\%), adormecimiento de un dedo $(25,24 \%)$ y el signo más prevalente fue dolor articular (12,62\%) (7).

Estudios han informado que el $66,3 \%$ de los odontólogos se encuentra en un rango de edad entre los 20 a 39 años y en su mayoría mujeres (61,4\%). En relación a las horas que labora el grupo en estudio, el 53\% lo realiza en un periodo de 8 o más horas, en cuanto a la antigüedad laboral, el $54 \%$ es menor a 9 años. Además, se ha comprobado que el $60 \%$ realiza su trabajo en una silla y unidad de trabajo ergonómicamente inadecuada. En conclusión, el $89 \%$ adquiere una postura incorrecta al realizar su práctica profesional, lo cual se refleja en dolor lumbar moderado en el $69 \%$ de los odontólogos, observándose que la edad es un factor que influye significativamente en su presencia (8). 
En relación a las condiciones de trabajo dadas por la utilización prolongada de equipos e instrumental odontológico en estudiantes de postgrado y en docentes, en Colombia se observó mayor sintomatología en cuello (62\%) seguida de hombros (47\%). La especialidad de endodoncia es la que presentó mayor sintomatología en la extremidad superior, y la zona anatómica más destacada la mano (83,3 \%). Las mujeres presentan mayor sintomatología en cuello $(74,1 \%)$, mientras que los hombres presentan mayores molestias en hombros (62,5\%). Estos datos son similares a lo reportado por otra investigación, que encontró dolor en cuello de 83,8\%, manos y muñecas $(73 \%)$ y región lumbar en un $81 \%(9,10)$.

El presente proyecto surge por la necesidad de identificar los problemas musculo esqueléticos en personal de odontología, debido a que el bienestar del profesional de la salud ocupa cada día un lugar más importante en la vida moderna, es por ello que el mayor nivel de información que se obtenga a través de las investigaciones sobre los problemas de salud ocupacional, contribuirá de manera especial a la promoción y prevención de las enfermedades y garantizar una mejor calidad de vida para el profesional (11). Donde enfermería, por sus competencias, puede desarrollar una labor fundamental, tanto en la pesquisa, como en intervenciones que permitan mantener una mejor salud laboral.

Por tanto, el objetivo general fue identificar TME en Odontólogos de una clínica dental en Hermosillo, Sonora. Los objetivos específicos fueron determinar las características sociodemográficas y nivel riesgo de TME que padecen los odontólogos en el estudio.

\section{> Material y método}

Estudio cuantitativo, no experimental y transversal, observacional. Los datos fueron recolectados du- rante el mes de abril del 2015. La unidad de análisis fueron odontólogos que se encontraban laborando en alguna de las nueve sucursales de una clínica dental privada de Hermosillo, Sonora, México.

La población objeto fueron 42 personas y la muestra estuvo constituida por 30 sujetos que aceptaron participar y que cumplían con los siguientes criterios de inclusión: a) presentar molestias en las regiones anatómicas de cuello, hombros, dorsal o lumbar, codos o antebrazos y muñecas o manos en los últimos 12 meses, b) estar laborando en el momento de la recolección de datos y c) aceptar participar firmando el consentimiento informado.

Se utilizó el Cuestionario Estandarizado Nórdico (12), validado en población mexicana, con Alfa de Cronbach de 0.83 , que muestra un esquema del cuerpo humano, con el propósito de que el entrevistado señale en qué parte de su cuerpo ha tenido dolor, molestias o problemas (13). Y el método Rapid Entire Body Assessment (REBA) que facilita una valoración rápida y sistemática del riesgo postural del cuerpo entero del trabajador (14). Este método clasifica la puntuación final en 5 rangos que se corresponden con un nivel de acción. Cada nivel de acción determina un nivel de riesgo y recomienda una actuación sobre la postura evaluada, señalando en cada caso la urgencia de la intervención. Cuanto mayor sea el valor del resultado, mayor será el riesgo previsto de la postura adoptada, es decir, el valor 1 indica un riesgo inapreciable, de 2-3 bajo, 4-7 medio, 8-10 alto, mientras que el 15 que es la puntuación máxima, destaca que se trata de una postura de riesgo muy alto, sobre la que se debe actuar de inmediato (15).

Además, se registraron variables sociodemográficas, como edad, sexo, mano dominante, años de ejercicio profesional, actividad odontológica profesional predominante y si presenta alguna condición o enfermedad. 
Las consideraciones éticas de esta investigación se apegaron a lo estipulado en la Declaración de Helsinki y al Reglamento de la Ley General de Salud en Materia de Investigación para la Salud de México $(16,17)$.

Para el análisis de los datos se utilizó el software IBM SPSS versión 20 y el Ergo/IBV V14 en versiones para Windows 8.1.

\section{) Resultados}

Respecto a las características sociodemográficas, la media edad de los sujetos fue de 31,6 años ( $D E=6,28$ años), la media de años que llevan ejerciendo la odontología fue de 8,53 años (DE=6.08).

TABLA 1. CARACTERÍSTICAS SOCIODEMOGRÁFICAS DE LOS PARTICIPANTES.

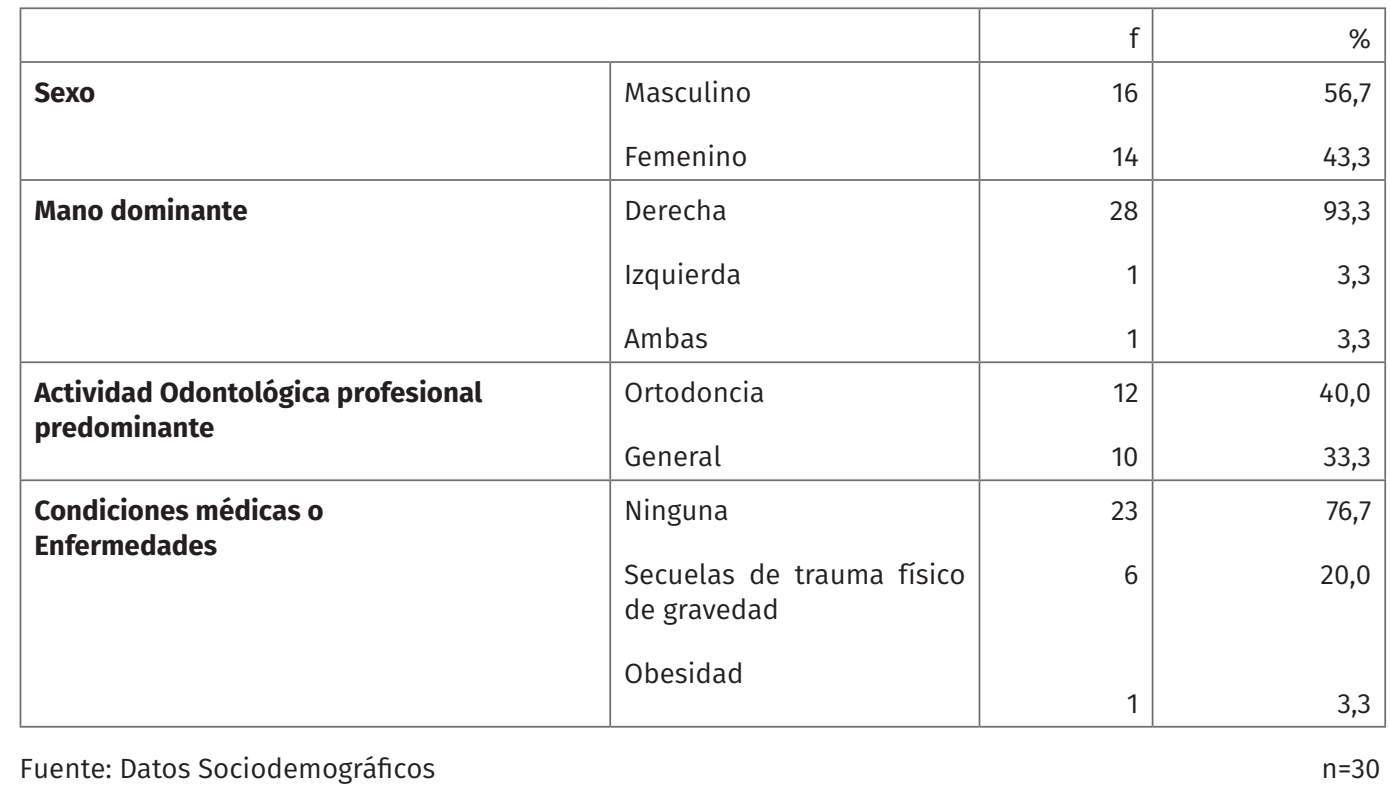

En la tabla 1 se muestra que el $56,7 \%$ de los participantes corresponde al sexo masculino, el 93,3\% responde que la mano predominante es la derecha, la actividad odontológica predominante es la ortodoncia $(40 \%)$ y en relación a la presencia de enfermedad, el 76,7\% no refiere ningún padecimiento o condición médica de importancia, mientras que $20 \%$ tiene secuelas de trauma físico de gravedad. 


\section{> Sintomatología músculo- esquelética}

TABLA 2. FRECUENCIA DE SINTOMATOLOGÍA MUSCULOESQUELÉTICA, IMPEDIMENTO PARA EL TRABAJO Y EL TRATAMIENTO RECIBIDO

\begin{tabular}{|c|c|c|c|c|c|c|c|c|}
\hline \multirow{3}{*}{$\begin{array}{l}\text { Región } \\
\text { anatómica }\end{array}$} & \multirow{2}{*}{\multicolumn{2}{|c|}{$\begin{array}{l}\text { Sintomatologia en los } 12 \\
\text { meses precedentes } \\
\text { f } \%\end{array}$}} & \multirow{2}{*}{\multicolumn{2}{|c|}{$\begin{array}{l}\text { Sintomatología en los } \\
7 \text { dias precedentes } \\
\\
\text { f } \%\end{array}$}} & \multirow{2}{*}{\multicolumn{2}{|c|}{$\begin{array}{l}\text { Impedimento para } \\
\text { el trabajo en los } 12 \\
\text { meses precedentes } \\
\text { f } \%\end{array}$}} & \multirow{2}{*}{\multicolumn{2}{|c|}{ 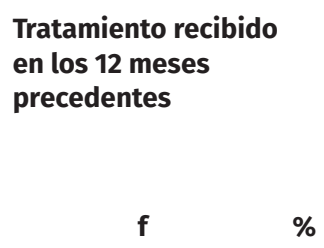 }} \\
\hline & & & & & & & & \\
\hline & 21 & 70,0 & 13 & 43,3 & 2 & 6,7 & 10 & 33,3 \\
\hline Hombro & 14 & 46,7 & 11 & 36,7 & 0 & 0,0 & 7 & 23,3 \\
\hline Dorsal o lumbar & 19 & 63,3 & 13 & 43,3 & 1 & 3,3 & 8 & 26,7 \\
\hline $\begin{array}{l}\text { Codo o } \\
\text { antebrazo }\end{array}$ & 3 & 10,0 & 2 & 6,7 & 0 & 0,0 & 1 & 3,3 \\
\hline Muñeca o mano & 14 & 46,7 & 8 & 26,7 & 0 & 0,0 & 5 & 16,7 \\
\hline
\end{tabular}

En la tabla 2, se puede observar la sintomatología músculo-esquelética presentada por los participantes, según región anatómica, donde la región más afectada en los últimos 12 meses corresponde al cuello (70\%) y, el codo o antebrazo derecho son la región menos afectada (10\%). En cuanto a la sintomatología en los últimos 7 días, se repiten las regiones con mayor y menor afección, siendo el cuello y zonas dorsal y lumbar las regiones con mayor sintomatología (43,3\%) y el codo el sitio con menor sintomatología (6,7\%).
Además, es importante señalar que debido a las molestias músculo-esqueléticas presentadas por los odontólogos, una cantidad importante debió cambiar su lugar de trabajo. Esta situación se produjo en el 23,3\% de los que presentaron molestias a nivel de cuello, el $10 \%$ por molestias en hombro, un $26,7 \%$ por molestias en la región dorsal o lumbar, un 3,3\% por molestias en codo o antebrazo y un $6,7 \%$ por molestias en muñeca y mano. 
TABLA 3. DURACIÓN DE LA SINTOMATOLOGÍA MUSCULO-ESQUELÉTICA SEGÚN REGIÓN ANATÓMICA.

\begin{tabular}{|c|c|c|c|c|c|c|c|c|c|}
\hline \multirow[t]{2}{*}{ Región anatómica } & \multicolumn{3}{|c|}{ Periodo de duración de la sintomatología } & \multicolumn{3}{|c|}{$\begin{array}{l}\text { Duración de sintomatología en los } 12 \\
\text { meses precedentes }\end{array}$} & \multicolumn{3}{|c|}{ Duración de cada episodio } \\
\hline & Duración & $\mathbf{f}$ & $\%$ & Duración & $\mathbf{f}$ & $\%$ & Duración & $\mathbf{f}$ & $\%$ \\
\hline \multirow[t]{2}{*}{ Cuello } & 1 Año & 4 & 13,3 & 1 a 7 Días & 9 & 30 & $1 \mathrm{a} 24 \mathrm{~h}$ & 6 & 20,0 \\
\hline & & & & Siempre & 6 & 20 & 1 a 7 Días & 6 & 20,0 \\
\hline \multirow[t]{2}{*}{ Hombro } & 1 Año & 3 & 10 & 1 a 7 Días & 5 & 16,7 & $1 \mathrm{a} 24 \mathrm{~h}$ & 4 & 13,3 \\
\hline & 2 Años & 3 & 10 & 8 a 30 Días & 5 & 16,7 & 1 a 7 Días & 4 & 13,3 \\
\hline \multirow[t]{2}{*}{ Dorsal o lumbar } & 1 Año & 4 & 13,3 & 1 a 7 Días & 5 & 16,7 & $<1 \mathrm{~h}$ & 6 & 20,0 \\
\hline & & & & Siempre & 7 & 23,3 & $1 \mathrm{a} 24 \mathrm{~h}$ & 6 & 20,0 \\
\hline \multirow[t]{4}{*}{ Codo o antebrazo } & 2 Meses & 1 & 3,3 & >30 Días, no Seguidos & 2 & 6,7 & 1 a 7 Días & 1 & 3,3 \\
\hline & 1 Año & 1 & 3,3 & & & & 1 a 4 Semanas & 1 & 3,3 \\
\hline & 2 Año & 1 & 3,3 & & & & $>1$ Mes & & \\
\hline & & & & & & & & 1 & 3,3 \\
\hline Muñeca o mano & 1 Año & 3 & 10,0 & 1 a 7 Días & 6 & 20,0 & 1 a 7 Días & 5 & 16,7 \\
\hline
\end{tabular}

En la tabla 3, destaca que, en cuanto al periodo de duración de la sintomatología por región, el hombro muestra los tiempos más prolongados entre 1 y 2 años (20\%) y el codo o antebrazo presenta simultáneamente los tiempos más extensos ( 2 años) y los más breves (2 meses) afectando al 9,9\% de los odontólogos.

En cuanto a las molestias en los 12 meses precedentes, se encontró existen profesionales que "siempre" mantienen las molestias, esta situación afecta al $20 \%$ de los odontólogos que padecen molestias en el cuello y el $23,3 \%$ de los que presentan molestias a nivel dorsal o lumbar.
Respecto a la duración de los episodios de las molestias, se observó una mayor frecuencia en los períodos que iban entre de 1 a 24 horas, para las regiones de cuello, hombro, dorsal lumbar y, episodios de 1 a 7 días en las regiones de cuello, hombro, codo o antebrazo y muñeca o mano.

Además, en una escala de 0 (sin molestias) a 5 (molestias muy fuertes), la mayoría calificó su molestia en 2 , de ellos un $20 \%$ refiere molestia en cuello, 16,7\% hombro, 23,3\% muñeca o mano. Además, un $20 \%$ califico su nivel de molestia en nivel 3, estas molestias correspondieron a la región dorsal o lumbar. 


\section{Valoración del riesgo postural}

TABLA 4. RIESGO POSTURAL DE LOS PARTICIPANTES

\begin{tabular}{|l|r|r|r|}
\hline & & $f$ & $\%$ \\
\hline Nivel de Acción & 2 & 21 & 70,0 \\
\hline Puntuación REBA & $4-7$ & 21 & 70,0 \\
\hline Nivel de riesgo & Medio & 21 & 70,0 \\
\hline Acción & Necesaria & 21 & 70,0 \\
\hline
\end{tabular}

Fuente: Evaluación mediante el Método REBA

$n=30$

En Tabla 4, se muestran los resultados de la aplicación del método REBA, se puede observar que el nivel de acción es 2 en el $70 \%$ de los casos, con la puntuación REBA está entre 4 y 7 un $70 \%$, esto quiere decir que los odontólogos encuestados muestran un nivel de riesgo medio (70\%) y en la misma proporción la realización de una intervención o acción es necesaria.

\section{> Discusión}

El propósito de esta investigación fue determinar los riesgos de TME en Odontólogos de una clínica dental en Hermosillo, Sonora. En el análisis de las características sociodemográficas se encontró un rango de edad entre 24 a 58 años, con una media de 31,6 años, en cuanto al sexo, más de la mitad pertenece al sexo masculino y el tiempo que llevan ejerciendo es en promedio de 8,53 años. Estos aspectos coinciden con lo reportado por otros autores en cuanto a edad y difiere en cuanto a sexo y antigüedad donde se informa una cantidad de años mayor (18), probablemente, esto se debe a las políticas internas actuales de la clínica dental en Hermosillo Sonora, donde se busca emplear odontólogos jóvenes, lo que hace que su antigüedad también sea menor.

Se observó que, de las ocho actividades odontológicas existentes, la ortodoncia es la actividad de mayor prevalencia (40\%). Contrastando con los datos proporcionados por una investigación ecuatoriana en el año 2012, donde 36\% realizan prioritariamente rehabilitación oral y operatoria y solo el $27 \%$ ortodoncia (4). Esta situación se articula, en parte, por el contexto de atención de las instituciones de salud gubernamentales, en donde los paquetes de atención poco atienden esta rama de la odontología y por ende, la población busca alternativas en diferentes espacios, como los privados, para tratar los problemas dento-bucales relacionados a ésta área.

En cuanto a la variable condiciones o enfermedades, la mayoría se encuentra en un adecuado estado de salud, sin embargo, existen odontólogos que tienen secuelas de trauma fisico de gravedad. Aspecto similar a otros estudios donde los padecimientos de artritis reumatoide, obesidad, secuela de trauma físico de gravedad y enfermedad neoplásica fueron escasamente reportadas (4).

Al evaluar la sintomatología de cada zona anatómica, cuando se realiza la práctica odontológica, se observó un predomino de sintomatología en cuello, seguido de región en dorsal o lumbar, casi la mitad expreso molestias de hombros con mayor molestia en lado derecho. También, menos de la mitad manifiesta tener molestias en manos o 
muñecas, con mayor frecuencia en lado derecho. En codos, el resultado fue escaso, ya que la mayoría generalmente señala no tener ninguna molestia. Comparado con los resultados de un estudio efectuado en Colombia en el 2014 (9), se observó que existe coincidencia en la sintomatología de cuello y hombros con mayor predominio en lado derecho, de la misma forma en codos fue donde menos se presentaron molestias (79\% no refirió la presencia de dolor en esta zona).

En cuanto a los periodos de permanencia de las molestias por región anatómica, los resultados variaron entre 2 años en hombro y codo o antebrazo, hasta 2 meses en codo o antebrazo. Contraponiéndose con los resultados de una investigación venezolana en 2014, que señaló una prevalencia de molestias osteomusculares en odontólogos, del 100\% durante los últimos 6 meses (18). Siendo el cuello y zona dorsal o lumbar donde las molestias se presentaban hace un año, solamente estos datos coinciden con los de mano o muñeca encontrados en esta investigación.

Como demuestran los datos procedentes, menos del $30 \%$ de los profesionales necesitó cambiar su puesto de trabajo a causa de molestias en zona dorsal o lumbar y en cuello. Según los resultados obtenidos por una investigación colombiana en el 2012, donde se analizó la posición de trabajo durante la práctica clínica de los estudiantes de odontología, se observó deficiente en un 43,1\%, lo cual indica un cambio urgente en el puesto de trabajo. Mientras que en el $34,8 \%$ se requiere un cambio rápido de postura (19). La comparación entre estudiante y profesionales es notoria, debido a que los estudiantes necesitaron cambiar de puesto en porcentaje mucho más alto.

Respecto a las molestias en los últimos 12 meses precedentes, prevalece la duración de 1 a 7 días en cuello y en hombro, además, la quinta parte de los encuestados siempre sufre molestias en dorsal o lumbar. Si comparamos con los datos obtenidos por otra investigación mexicana realizada en el 2009, el $90 \%$ reporta dolor en espalda baja por más de un mes y el $80 \%$ en alguna ocasión tuvo ese dolor, el $100 \%$ tuvo dolor en cuello y hombros en el último año, el 70\% tuvo reducción de su actividad por dolor de cuello y el $80 \%$ por dolor de hombro (20), observándose diferencias porcentuales importantes, tanto en la zona anatómica como en la duración de la molestia en 12 meses precedentes.

En cuanto a la duración de los episodios, se mostró que cuello y hombro mostraban una persistencia de 1 hora hasta los 7 días cada uno y en zona dorsal o lumbar el tiempo fue menor a una hora. Cotejando esto con lo mostrado en el estudio hecho en Venezuela en 2014, los padecimientos más frecuentes en los odontólogos por duración de siete días, fueron en las zonas: del cuello $58 \%$, parte superior espalda 51,8 \%, hombros $44,4 \%$ y en parte baja espalda 43,2 \% (21). Por lo tanto, es posible señalar que hubo relación, en las zonas corporales mencionadas, pero en este estudio los porcentajes observados en el periodo de 1-7 días en las regiones dorsal o lumbar, son menores.

El tiempo que las molestias, han impedido que los odontólogos puedan hacer su trabajo en el último año es reducido, ya que un bajo porcentaje ha estado incapacitado para laborar por molestias de cuello, dorsal o lumbar; en las demás zonas no se presentaron molestias que les impidieran trabajar. Los aspectos señalados concuerdan con los resultados de una tesis de investigación realizada con estudiantes de odontología de Colombia en 2012, donde el 18,8\% indicó que no representaba ningún obstáculo para su práctica clínica, el 63,8\% indicó que puede hacer su trabajo, pero le causa algunos síntomas y ninguno de los participantes indicó estar incapacitado para realizar su trabajo a causa del 
dolor o molestia relacionado a la práctica clínica (22). Probablemente, las incapacidades mostradas por los odontólogos de este estudio, se deba a que el tiempo dedicado a su actividad práctica es mayor que el realizado por los estudiantes, quienes pasan menos tiempo en sus actividades odontológicas.

El tratamiento recibido en los últimos 12 meses, fue a causa de molestias en cuello principalmente, seguido por la zona dorsal o lumbar y hombro. En la investigación venezolana del 2014, se observó que el $66 \%$ ha recibido tratamiento en los últimos 3 meses (18). Apreciando divergencias con lo encontrado en esta investigación, donde existió mayor necesidad de tratamiento en un periodo más corto de tiempo.

Las molestias en los últimos 7 días revelan que en cuello y región dorsal o lumbar son las zonas anatómicas donde más se han presentado molestias en la última semana. En los resultados de investigadores mexicanos en el 2009 , el $80 \%$ de las mujeres presentó dolor en la parte superior de la espalda durante los últimos 7 días, en contraste con el $40 \%$ de los hombres (20), estos resultados se aproximan bastante a lo presentado en los odontólogos de Hermosillo.

Las molestias atribuidas a la posición, tienen un papel relevante en la mayoría de las partes anatómicas, siendo predominante en cuello y en dorsal o lumbar. Según los resultados pertenecientes la investigación colombiana de 2012, encontraron que algunas prácticas y posturas inadecuadas relacionadas con la práctica clínica odontológica se encuentran directamente relacionadas con las alteraciones osteomusculares de los participantes (19). Lo que es concordante con lo mostrado por esta investigación donde la posición y la carga laboral en ocasiones excesiva, tienen un papel importante en las molestias reportadas.
Al evaluar los resultados provenientes del método REBA, la mayoría los odontólogos obtuvo una puntuación de 4 a 7, lo que muestra un nivel de riesgo medio y requieren una acción o intervención necesaria. Aspecto que contrasta con lo reportado por un estudio argentino del 2015, donde se realizó una investigación en estudiantes de odontología, aquí el 53\% presentó un nivel de riesgo máximo, requiriendo de una actuación inmediata, para modificar posturas incorrectas, el $42 \%$ presentó un nivel de riesgo alto por lo que requerían una pronta intervención y solo el $5 \%$ se encontró en un nivel de riesgo medio pero igual precisa de una intervención correctiva (5). Al confrontar ambas investigaciones los estudiantes presentaron niveles de riesgo máximo, y los profesionales mostraron un nivel medio, esto podría deberse a la experiencia profesional y conocimientos aplicados en su trabajo.

Con todo lo anterior es posible concluir que las principales molestias reportadas por odontólogos de este estudio fueron en cuello, región dorsal y lumbar. El riesgo de TME mediante el método REBA es medio, el cual amerita una acción necesaria. Por lo tanto, es necesaria un área ergonómicamente correcta para desarrollar su actividad con las mínimas repercusiones de salud a largo plazo.

Como sugerencias, se pueden señalar que, en cuanto las instalaciones, se observó que no en todos los consultorios poseen el espacio suficiente para movimiento de esfera de reloj, necesario en el trabajo del odontólogo, esto posibilita los cambios de posturas durante su jornada laboral, por otra parte, no todos emplean sillas adecuadas. Los siguientes aspectos deben ser considerados en el diseño ergonómico del puesto de trabajo en una clínica odontológica: silla, altura, ángulos de confort, espacios disponibles, superficies de apoyo, distancias y ángulos visuales, zona de alcance óptimo, dimensiones del puesto de trabajo (23). 
A los odontólogos se les sugiere, realizar acciones destinadas a mejorar su postura durante su actividad odontológica. Por ejemplo, evitar la inclinación de la cabeza, encoger los hombros y trabajar inclinado, ya que producen tensión muscular. No hacer giros o movimientos laterales, ya que hacen que la columna vertebral esté forzada, por lo que se recomienda trabajar con la espalda lo más recta posible. Y durante el descanso es preferible cambiar de postura y alejarse del puesto de trabajo $(24,25)$. Aspectos que podrían incorporarse en una capacitación donde participe un equipo multidisciplinario que incluya profesionales de enfermería.

\section{Referencias bibliográficas}

1. Beltrán M. De lo oral a lo bucal en la odontología. Revista Colombiana de Investigación en Odontología. [Internet]. 2011 [Citado el 19 ene 2016]; 2(5). Disponible en: http://www.rcio.org/index.php/rcio/article/view/58/140.

2 Barrancos J, Barrancos P. Operatoria dental: integración clínica. 4ta ed. Buenos Aires. Argentina: Médica Panamericana; 2006.

3. Organización Mundial de la salud (OMS). Factores de riesgo [Internet]. OMS; 2015 [Citado el 15 abr 2015]. Disponible en: http://www.who.int/ topics/risk_factors/es/

4. Mieles P. Ergonomía dental y su incidencia en las complicaciones musculoesqueléticas en odontólogos de la ciudad de Portoviejo. [Tesis]. Ecuador: Universidad de San Gregorio de Portoviejo, 2012.

5. Jacome NC, Gigena PC. Estrategia de intervención para disminuir el riesgo postural en estudiantes de odontología durante la atención clínica. Universidad Nacional de Córdoba. Argentina. Huellas: revista de vinculación con la comunidad de la Facultad de Odontología. [Internet]. 2014 [Citado el 19 ene 2016]; 2(4). Disponible en: https//
revistas.unc.edu.ar/index.php/HUELLAS/article/ view/77603/8571

6. Guerra R. Las lesiones músculo esqueléticas en el área ocupacional de la odontología. Caso: odontólogos docentes de la facultad de odontología de la Universidad José Antonio Páez. La pasión del saber. [Internet] 2011. [Citado el 23 mar 2015]; (3) Disponible en: http://www.ujap.edu.ve/pasion/ index2.php?option=com_content\&do_pdf=1\&id=99

7. Fortich N. Prevalencia de signos y síntomas de trastornos de la mano en profesionales odontólogos de la ciudad de Cartagena en el 2012. [Tesis de Maestría]. Colombia: Corporación Universitaria Rafael Núñez, 2012.

8. García E, Noriega K. Asociación entre dolor lumbar y postura de trabajo durante la práctica profesional del cirujano dentista en la ciudad de Toluca. [Tesis de Pregrado] México: Universidad Autónoma del Estado de México, 2013.

9. Sánchez K, Rodríguez M, Gutiérrez A, Trujillo L, Ramírez L, Mireya E. Condiciones de trabajo relacionados con desórdenes musculoesqueléticos de la extremidad superior en residentes de odontología, Universidad El Bosque Bogotá, D.C. (Colombia). Salud Uninorte. [Internet] 2014 [Citado el 23 mar 2015]; 30 (1). Disponible en: http://rcientificas.uninorte. edu.co/index.php/salud/article/view/5814/4765

10. Mora L. Caracterización de los factores de riesgo de desorden musculoesquelético asociados a las condiciones de la tarea. Caso: clínica odontológica adulto I. [Tesis de magíster]. Bogotá: Universidad Nacional de Colombia, 2014.

11. Briones A. Posturas odontológicas ergonómicas y dolor muscular, durante las prácticas clínicas del estudiante del 5to año de la facultad de odontología periodo 2013. [Internet] [Tesis de Pregrado]. Guayaquil: Universidad de Guayaquil, 2014.

12. Ministerio de Salud de Chile (MIN$\mathrm{SAL}$ ). Protocolos de vigilancia para trabajado- 
res expuestos a factores de riesgo de trastornos musculoesqueléticos de extremidades superiores relacionados con el trabajo. [Internet]. Santiago de Chile; 2012 [Citado el 13 mar 2015]: Disponible en: http://web.minsal.cl/portal/url/item/dbd6275dd3c8a29de040010164011886.pdf.

13. Montoya M, Palucci M, Cruz M, Taubert de Freitas F. Lesiones osteomusculares en trabajadores de un hospital mexicano y la ocurrencia del ausentismo. Cienc enferm. 2010; XVI(2): 35-46.

14. Instituto Nacional de Seguridad e Higiene en el Trabajo de España (INSHT). NTP 601: Evaluación de las condiciones de trabajo: carga postural. Método REBA (Rapid Entire Body Assessment). España; 2001. [Citado el 18 abr 2015]. Disponible en: http://www.insht.es/InshtWeb/ Contenidos/Documentacion/FichasTecnicas/NTP/ Ficheros/601a700/ntp_601.pdf

15. Instituto Nacional de Seguridad e Higiene en el Trabajo de España (INSHT). Análisis de posturas forzadas [Internet]. España; 2015. [Citado el 18 de Abr 2015]. Disponible en: http://calculadores. insht.es:86/An\%C3\%A1lisisdeposturasforzadas/ Introducci\%C3\%B3n.aspx

16. Declaración de Helsinki de la Asociación Médica Mundial (AMM). Principios éticos para las investigaciones médicas en seres humanos. [Internet] 2013 [Citado el 13 de Abr del 2015]. Disponible en: http://www.wma.net/ es/30publications/10policies/b3/

17. Secretaria de Salud de México. Reglamento de la Ley General de Salud en Materia de Investigación para la Salud. [Internet] 2012 [Citado el 14 abr 2015]. Disponible en: http://www.salud.gob. $\mathrm{mx} /$ unidades/cdi/nom/compi/rlgsmis.html.

18. Ancheta E. Caracterización de los trastornos musculo esqueléticos en profesionales de odontología, municipio de Girardot, Estado Aragua. [Proyecto de especialidad] Venezuela: Univer- sidad de Carabobo, 2014

19. Fals J, González F, Orozco J, Correal S, Perne $C$. Alteraciones osteomusculares asociadas a factores físicos y ambientales en estudiantes de odontología. Rev bras epidemiol. 2012; 15(4): 88495.

20. Chávez R, Preciado M, Colunga C, Mendoza $P$, Aranda C. Trastornos Músculo-esqueléticos en Odontólogos de una Institución Pública de Guadalajara, México. Cienc Trab. (jul-sep) 2009; 11 (31): 152-5.

21. Angarita, A, Castañeda A, Villegas E, Soto M. Revisión sistemática sobre enfermedades laborales en odontología. Act Biocl. [Internet] 2014. [Citado el 23 de Abr 2015]; Disponible en: http:// erevistas.saber.ula.ve/index.php/actabioclinica/ article/view/4962.

22. Ocampo N. Riesgo Ergonómico en Estudiantes de Odontología de la Universidad de Antioquia, Colombia. [Tesis de Maestría]. Colombia: Universidad Pompeu Fabra, 2012.

23. Islam E. Relación de las técnicas ergonómicas con la práctica clínica de la Facultad de Odontología. Diseño de protocolo ergonómico. [Tesis de Pregrado]. Guayaquil: Universidad de Guayaquil, 2014.

24. García E, Noriega K. Asociación entre dolor lumbar y postura de trabajo durante la práctica profesional del cirujano dentista en la ciudad de Toluca. [Tesis] México: Universidad Autónoma del Estado de México, 2013.

25. Comisiones Obreras de Castilla y Nueva León, Secretaria de Salud Laboral de castilla y Nueva León. Manual de trastornos musculoesqueléticos.; 2008 [Citado el 3 may 2015]. Disponible en: http:/ / www.castillayleon.ccoo.es/comunes/recursos/6/doc11488_Manual_de_Trastornos_Musculoesqueleticos_\%282_edicion._2010\%29.pdf. 